\title{
First-principles analysis of electron-phonon interactions in graphene
}

\author{
K. M. Borysenko, ${ }^{1}$ J. T. Mullen, ${ }^{2}$ E. A. Barry, ${ }^{1}$ S. Paul, ${ }^{2}$ Y. G. Semenov, ${ }^{1}$ J. M. Zavada, ${ }^{1}$ \\ M. Buongiorno Nardelli, 2,3 and K. W. Kim ${ }^{1, *}$ \\ ${ }^{1}$ Department of Electrical and Computer Engineering, North Carolina State University, Raleigh, North Carolina 27695-7911, USA \\ ${ }^{2}$ Department of Physics, North Carolina State University, Raleigh, North Carolina 27695-8202, USA \\ ${ }^{3}$ CSMD, Oak Ridge National Laboratory, Oak Ridge, Tennessee 37831, USA
}

(Received 9 February 2010; published 16 March 2010)

\begin{abstract}
The electron-phonon interaction in monolayer graphene is investigated using density-functional perturbation theory. The results indicate that the electron-phonon interaction strength is of comparable magnitude for all four in-plane phonon branches and must be considered simultaneously. Moreover, the calculated scattering rates suggest an acoustic-phonon contribution that is much weaker than previously thought, revealing an important role of optical phonons even at low energies. Accordingly it is predicted, in good agreement with a recent measurement, that the intrinsic mobility of graphene may be more than an order of magnitude larger than the already high values reported in suspended samples.
\end{abstract}

DOI: 10.1103/PhysRevB.81.121412

PACS number(s): 72.10.Di, 71.15.Mb, 72.80.Vp

Graphene, a two-dimensional (2D) sheet of carbon atoms in a honeycomb lattice, continues to attract much attention due to its unique physical properties. Aside from a substantial academic interest resulting from the relativisticlike behavior of charge carriers, this material is considered very promising in device applications as it has an extremely high intrinsic mobility, even at room temperature. Although in realistic conditions (i.e., placed on a substrate) the mobility tends to decrease significantly due to the presence of additional scattering mechanisms at the interfaces,,${ }^{1-3}$ much effort is currently being devoted to eliminate, or at least minimize, these effects which are detrimental to graphene transport characteristics. Therefore, it is crucial to develop an accurate knowledge of the electron-phonon scattering as it determines the ultimate limit of any electronic device performance. The strength of electron-phonon coupling is typically estimated using the deformation potential approximation (DPA); it has been applied for graphene by a number of authors. ${ }^{4-6}$ When the corresponding deformation potential constant was estimated from the transport measurements, however, the results revealed a discrepancy that is too large to be ignored. ${ }^{1,2,7}$ Moreover, a very recent observation of mobilities in excess of $10^{7} \mathrm{~cm}^{2} / \mathrm{V} \mathrm{s}$ at $T \lesssim 50 \mathrm{~K}$ in the decoupled graphene 8 drastically departs from the conventionally accepted values, raising serious questions about the current understanding of the intrinsic transport characteristics of graphene. A detailed theoretical analysis of electron-phonon interaction beyond the DPA is clearly called for.

In this work, we apply a first-principles approach based on density-functional theory (DFT) to calculate the electronphonon coupling strength in graphene. The obtained electron-scattering rates associated with all phonon modes are analyzed and the intrinsic resistivity and mobility of monolayer graphene are estimated as functions of temperature. The results clearly elucidate the role of different branches (particularly, the significance of optical phonons and intervalley scattering via acoustic phonons) as well as limitations of DPA. The obtained effective deformation potential constants suggest the possibility that the intrinsic scattering rates may presently be overestimated, potentially due to the complex influence of extrinsic mechanisms, including the substrate. A subsequent Monte Carlo calculation predicts, in good agreement with Ref. 8, that the mobility of graphene could be more than an order of magnitude larger than the already high values reported in suspended samples ${ }^{1}$ when presumably all of the extrinsic scattering sources are eliminated.

A key component of our theoretical study is the evaluation of the electron-phonon interaction matrix elements by employing density-functional perturbation theory (DFPT) within the DFT formalism. ${ }^{9}$ This technique has the advantage of dealing with arbitrary electronic state $\mathbf{k}$ and phononic state $\mathbf{q}$ on an equal footing. Each phonon is treated as a perturbation of the self-consistent potential created by all electrons and ions. The calculation of the potential change due to this perturbation gives the value of the electronphonon matrix element,

$$
g_{\mathbf{k}+\mathbf{q}, \mathbf{k}}^{(i, j) \nu}=\sqrt{\frac{\hbar}{2 M \omega_{\nu, \mathbf{q}}}}\left\langle j, \mathbf{k}+\mathbf{q}\left|\Delta V_{\mathbf{q}, S C F}^{\nu}\right| i, \mathbf{k}\right\rangle,
$$

where $|i, \mathbf{k}\rangle$ is the Bloch electron eigenstate with the wave vector $\mathbf{k}$, band index $i$, and energy $E_{i, \mathbf{k}} ; \Delta V_{\mathbf{q}, S C F}^{\nu}$ is the derivative of the self-consistent Kohn-Sham potential ${ }^{9}$ with respect to atomic displacement associated with the phonon from the branch $\nu$ with the wave vector $\mathbf{q}$ and frequency $\omega_{\nu, \mathbf{q}}$; and $M$ is the atomic mass. A similar calculation has previously been performed in graphene for $\mathbf{q}$ at highsymmetry points $K$ and $\Gamma$, where Kohn anomalies occur (see below). ${ }^{10,11}$ However, the scattering rate calculation requires summation over the entire first Brillouin zone. Using DFT and DFPT (QUANTUM-ESPRESSO ${ }^{12}$ ), the electronic band structure and the phonon dispersion are calculated for a single graphene sheet in vacuum. The computation is performed on a hexagonal unit cell with the two-atom basis, lattice constant $a$ of $2.42 \AA$, and approximately $5 \AA$ of vacuum space, based on a norm-conserving pseudopotential and the generalized gradient approximation or local-density approximation. ${ }^{13}$ A Monkhorst-Pack grid ${ }^{14}$ of $36 \times 36 \times 1$ is used with no offset for integration that covers the first Bril- 

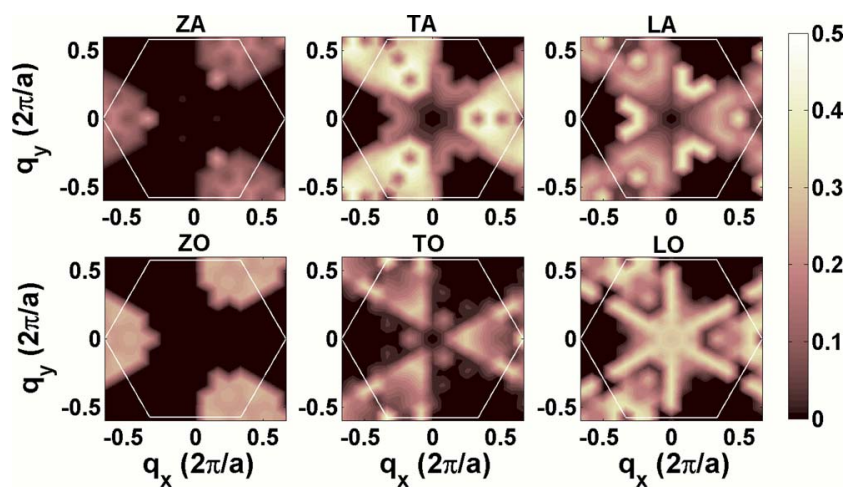

FIG. 1. (Color online) Electron-phonon interaction matrix elements $\left|g_{\mathbf{k}+\mathbf{q}, \mathbf{k}}^{(i, j)}\right|$ (in units of eV) calculated by DFPT for $\mathbf{k}$ at the conduction-band minimum (i.e., the Dirac point) as a function of phonon wave vector q. High anisotropy beyond the longwavelength approximation is clearly visible. The presence of Kohn anomalies reported earlier is revealed as the peaks (light color) at three equivalent Dirac points for TO and at the center of the Brillouin zone for LO.

louin zone and includes the points of high symmetry: $\Gamma, K$, and $M$.

Figure 1 shows the resulting matrix elements for an electron at the Dirac point [i.e., $\mathbf{k}=(4 \pi / 3 a, 0)]$ as a function of the phonon wave vector $\mathbf{q}$. The contribution of all six phonon branches is considered, whereas the electronic states (both initial and final) are limited to the lowest conduction band. The matrix elements display a pronounced anisotropy everywhere except near the Brillouin-zone center $(\mathbf{q}=0)$, where they are in qualitative agreement with the DPA (i.e., nearly isotropic) and also comply with the group theory analysis of electron-phonon interaction in graphene (in-plane modes): ${ }^{15}$ $\lim _{q \rightarrow 0}\left|g^{a c}\right| \sim q$ and $\lim _{q \rightarrow 0}\left|g^{o p}\right| \sim$ const for acoustic and optical phonons, respectively. Another important feature is the Kohn anomaly - a strong coupling of the electrons with inplane optical-phonon branches in points of high symmetry. ${ }^{10}$ This is prominently illustrated by the three sharp peaks at three equivalent $K$ points in the TO mode. The other Kohn anomaly (LO branch, $\mathbf{q}$ at the $\Gamma$ point) is not as distinct. Even more crucial is that their magnitude can be directly validated against experimental measurements. Comparison with available experiments and another $a b$ initio approach (GW) (Ref. 16) clearly shows good agreement, although DFT is known to underestimate the electron exchangecorrelation energy in the presence of Kohn anomaly. ${ }^{11}$ Specifically, the GW calculation and the experiments (Raman spectroscopy and inelastic x-ray scattering), as discussed in Ref. 11 and the references therein, provide values for $|g|^{2}$ that are larger than our results by about $15 \%$ and $60 \%$ for $\mathbf{q}$ at the $\Gamma$ (LO) and $K$ (TO) points, respectively. This discrepancy, however, is appreciable only in a very small portion of the first Brillouin zone and does not lead to a substantial difference when the electron interaction with the entire phonon system is taken into account. Accordingly, the scattering matrix elements obtained by DFPT are expected to provide sufficient accuracy to calculate the intrinsic transport properties of monolayer graphene.

Once the matrix elements are determined, the correspond-
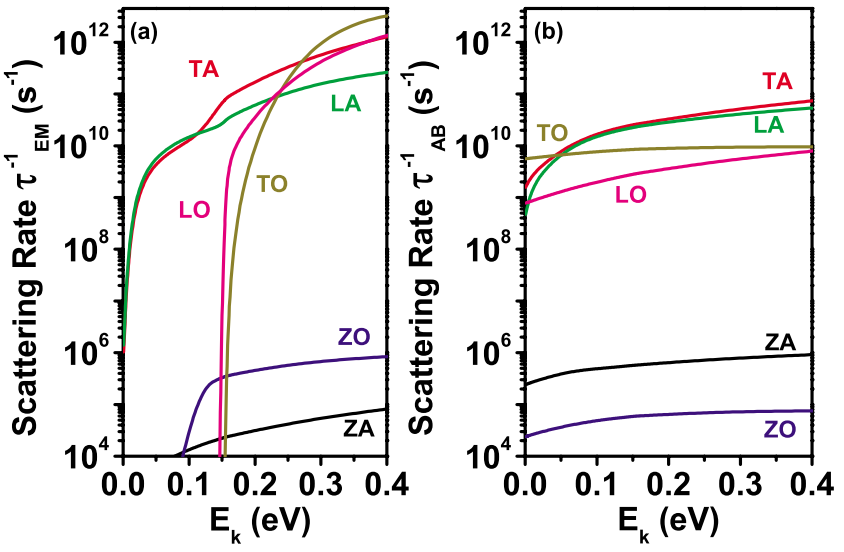

FIG. 2. (Color online) Phonon (a) emission and (b) absorption scattering rates at $T=300 \mathrm{~K}$ as functions of electron energy $E_{\mathbf{k}}$, as k changes along the $K-\Gamma$ direction in monolayer graphene. The contributions of all six branches are shown separately. The out-ofplane phonons (ZA and $\mathrm{ZO}$ ) do not play an important role. At very low electron energies $\left(E<k_{B} T\right)$, the scattering with absorption of TO phonons is dominant.

ing electron-phonon scattering rate can be obtained by using the Fermi's golden rule. As is the case of transition probability per unit time, our formulation assumes that all final electronic states are available (i.e., no dependence on the carrier distribution function). Figure 2 shows the computed outcome at room temperature $(T=300 \mathrm{~K})$ based on the DFPT data described above [Eq. (1)]. Although the result is plotted specifically for electrons with wave vector $\mathbf{k}$ along the $K-\Gamma$ direction, its directional dependence is minor in the energy range under consideration. The total electron-scattering rate (from all six branches) for $E \sim k_{B} T$ is on the order of $10^{10} \mathrm{~s}^{-1}$. This is lower than one might expect based on earlier estimates; $;^{2,4,6}$ a discussion on the possible reasons is given below in connection with Fig. 3. More specifically, the scattering rates for the out-of-plane phonons (acoustic ZA and optical ZO modes) are much smaller (at least three orders of magnitude) in comparison with the rest of the branches (in-plane modes). In 2D structures with an in-plane reflection symmetry (e.g., graphene), only in-plane phonon modes can couple linearly to electrons. ${ }^{15}$ On the other hand, all four in-plane branches contribute comparable scattering rates at room temperature despite the differences in phonon dispersion. Accordingly, optical phonons can play an important role even at low electron energies. Another interesting feature is a change in slope in the emission rates of TA and LA phonons [Fig. 2(a)]. This occurs due to the onset of zoneedge phonon emission near the $K$ points (denoted as $\mathbf{q}=K$ for simplicity) leading to intervalley transfer of electrons. As shown, intervalley scattering by TA phonons can be important since the emission threshold is relatively low: $\hbar \omega_{K}^{T A}$ $\simeq 120 \mathrm{meV}$ is substantially smaller than the corresponding TO and LO phonon energies.

The obtained scattering rates can be used to predict the intrinsic transport properties of graphene. The temperature dependence of the electrical resistivity is of particular interest as it is believed to be relatively insensitive to the impact of environment (e.g., substrate), ${ }^{2}$ potentially enabling a direct 


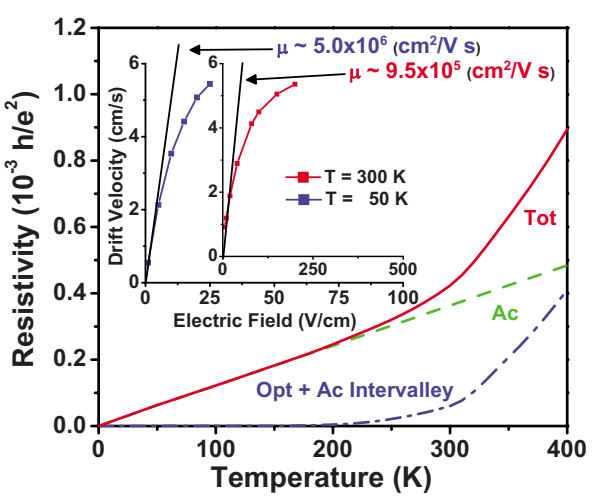

FIG. 3. (Color online) Intrinsic resistivity as a function of temperature. At $T<200 \mathrm{~K}$, the quasielastic intravalley scattering with in-plane acoustic phonons is dominant (dashed line). At higher temperatures, the contributions of both optical phonons and intervalley scattering by TA and LA modes (dashed-dotted line) lead to an exponential growth of the resistivity. The inset shows the low-field mobilities at $T=50$ and $300 \mathrm{~K}$, as determined from the slope of the drift-velocity versus electric field characteristic obtained by a fullband Monte Carlo simulation.

comparison with measurements in real (nonideal) samples. Following the relaxation-time approximation, ${ }^{17}$ an estimate of the intrinsic resistivity can be expressed straightforwardly $\mathrm{as}^{4}$

$$
\rho_{i} \approx\left[e^{2} D\left(E_{F}\right) \frac{v_{F}}{2} \tau_{t o t}\left(E_{F}\right)\right]^{-1},
$$

where $D\left(E_{F}\right)$ is the electronic density of states at the Fermi level $E_{F}, \tau_{\text {tot }}^{-1}\left(E_{F}\right)$ denotes the total scattering rate at $E_{F}$, and $v_{F}=10^{8} \mathrm{~cm} / \mathrm{s}$ is the Fermi velocity. Figure 3 shows the result of this calculation for $n=10^{12} \mathrm{~cm}^{-2}$. At relatively low temperatures $(T<200 \mathrm{~K})$, the electrons mostly scatter quasielastically with TA and LA phonons (intravalley scattering), leading to a linear temperature dependence of resistivity. As the temperature increases $(T>200 \mathrm{~K})$, the contributions of both optical phonons and intervalley scattering by TA and LA modes increase. The exponential slope in this region signifies the sensitivity of large-energy phonon occupancy to temperature.

Within the DPA, the slope of the intrinsic resistivity in the linear region is proportional to the square of the acousticphonon deformation potential constant. ${ }^{4}$ Using the DFPTbased results, we estimate a value of the effective deformation potential constant to be $D_{a c} \simeq 4.5 \mathrm{eV}$ for the combined contribution of TA and LA phonons. When compared to those extracted from the experiments, this number is fairly close to the recently reported $7.8 \mathrm{eV},{ }^{7}$ while much smaller than others $(29$ and $18 \mathrm{eV}) .{ }^{1,2}$ On the theory side, however, $D_{a c}$ of similar magnitude $(2.6 \mathrm{eV})$ was also deduced from a valence force model. ${ }^{18}$ With the experimental estimates under nonintrinsic conditions consistently larger than the intrinsic theoretical prediction, one possible explanation is that the influence of the substrate on graphene electron transport may currently be underestimated. This is the most apparent interpretation of the wide spread between experimental results
(7.8-29 eV) indicative of additional factors or mechanisms in play.

The issue of identifying intrinsic scattering characteristics is further examined by comparing our calculations with the results in the decoupled graphene layers (allegedly, the purest form of graphene), where the electron mobilities in excess of $10^{7} \mathrm{~cm}^{2} / \mathrm{V} \mathrm{s}$ were obtained from measurements at low temperatures $T \lesssim 50 \mathrm{~K} .{ }^{8}$ For this, a full-band Monte Carlo simulation is performed. ${ }^{19}$ The model utilizes the complete electron and phonon spectra in the first Brillouin zone. The electron-phonon interaction by four dominant in-plane modes, as described above by DFPT, is taken into account including the final-state selection after scattering. The inset in Fig. 3 shows the obtained velocity-field curves, where the low-field mobility is estimated from the slope in the linear region. The calculation produces a very high number of approximately $5 \times 10^{6} \mathrm{~cm}^{2} / \mathrm{V} \mathrm{s}$ at $50 \mathrm{~K}$, in good agreement with Ref. 8. Accordingly, the results clearly indicate the accuracy of the DFPT scattering rates, particularly those by TA and LA phonons, as the low-field mobility in this temperature range is dominated by the interactions with acoustic modes (see Fig. 3). Note that the calculated strength of the electron-optical-phonon interaction was validated earlier in connection with the Kohn anomaly. A corresponding estimation at $300 \mathrm{~K}$ predicts the intrinsic mobility approaching $10^{6} \mathrm{~cm}^{2} / \mathrm{V} \mathrm{s}$ that is also much higher than previously thought.

For practical application, it would be convenient to approximate the $a b$ initio electron-scattering rates by a simple analytical model. Presently, the expression commonly used for the acoustic-phonon scattering is given as ${ }^{4}$

$$
\left(\frac{1}{\tau_{\mathbf{k}}}\right)_{a c}=\left(\frac{k_{B}}{4 \hbar^{3} v_{F}^{2} \rho_{m} v_{s}^{2}}\right) D_{a c}^{2} T E_{\mathbf{k}},
$$

while

$$
\begin{aligned}
\left(\frac{1}{\tau_{\mathbf{k}}}\right)_{o p}= & \frac{D_{0}^{2}}{\rho_{m} \omega_{0}\left(\hbar v_{F}\right)^{2}}\left[\left(E_{\mathbf{k}}-\hbar \omega_{0}\right)\left(N_{\mathbf{q}}+1\right) \Theta\left(E_{\mathbf{k}}-\hbar \omega_{0}\right)\right. \\
& \left.+\left(E_{\mathbf{k}}+\hbar \omega_{0}\right) N_{\mathbf{q}}\right]
\end{aligned}
$$

is adopted for the optical-phonon scattering. ${ }^{6}$ Here, $v_{s}$ de-

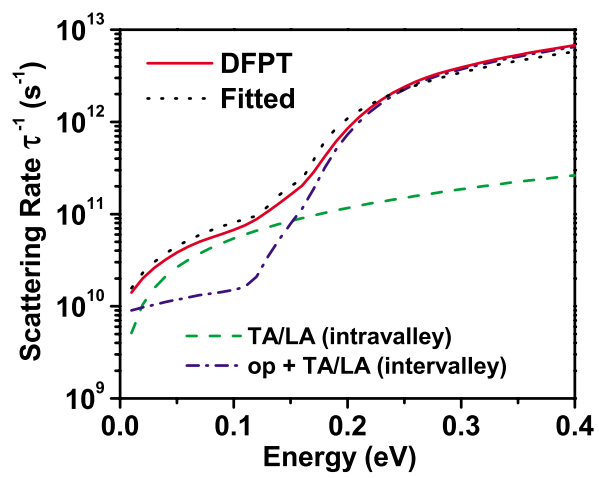

FIG. 4. (Color online) Total scattering rate as a function of electron energy at $T=300 \mathrm{~K}$. The dotted line shows the fitted curve calculated from Eq. (5). Clearly, the role of optical-phonon scattering and intervalley transfer must be taken into account in most cases judging from the discrepancy between the solid (total) and dashed (TA and LA intravalley) lines. 
notes the sound velocity, $\rho_{m}$ is the mass density, $N_{\mathbf{q}}$ is the phonon occupation number, and $\Theta(x)$ is the Heaviside step function. Although these formulas are valid under limited conditions [for example, Eq. (3) takes into account only the LA phonons in the long-wave approximation], the deformation potential constants $D_{a c}$ and $D_{0}$ can be treated as effective quantities to determine the contribution of all the relevant modes. Then, the total rate can be represented as a sum,

$$
(1 / \tau)_{t o t}=(1 / \tau)_{a c, K K}+(1 / \tau)_{a c, K K^{\prime}}+(1 / \tau)_{o p}
$$

Of the combined contribution of TA and LA phonons, the first term $(1 / \tau)_{a c, K K}$ represents electron intravalley scattering described by Eq. (3), whereas the intervalley transfer $(1 / \tau)_{a c, K K^{\prime}}$ is calculated by Eq. (4) since there is no distinction between acoustic and optical modes near the zone edge. The last term accounts for the interactions with both TO and LO modes. Figure 4 shows the results with the following parameters at $300 \mathrm{~K}:(1 / \tau)_{a c, K K}-D_{a c}=4.5 \mathrm{eV} ;(1 / \tau)_{a c, K K^{\prime}}$ $-D_{0}=3.5 \times 10^{8} \mathrm{eV} / \mathrm{cm}, \quad \hbar \omega_{0}=\hbar \omega_{K}^{T A}=124 \mathrm{meV}$; and $(1 / \tau)_{o p}-D_{0}=1 \times 10^{9} \mathrm{eV} / \mathrm{cm}, \quad \hbar \omega_{0}=\hbar \omega_{K}^{T O}=164.6 \mathrm{meV}$. Clearly, Eq. (5) not only serves as an excellent analytical approximation but also reveals the interplay among different scattering processes at a given temperature and electron energy. This simple model is expected to remain valid for $T$ $>T_{B G}$ with the Bloch-Grüneisen temperature $T_{B G}$ $\approx 50-60 \mathrm{~K}^{4}$

In summary, our first-principles analysis clearly illustrates that all in-plane phonons play an important role in electronphonon interactions in graphene and must be considered for transport studies at room temperature. Moreover, the results suggest that the influence of the substrate may be more significant than previously understood, clarifying at least in part the discrepancies observed in the strength of electronacoustic-phonon scattering. Under ideal conditions, it is predicted that the mobility of graphene could reach as high as $10^{6} \mathrm{~cm}^{2} / \mathrm{V} \mathrm{s}$ at room temperature.

This work was supported, in part, by the DARPA/HRL CERA, NSF, and FCRP FENA programs. M.B.N. wishes to acknowledge partial support from the Office of Basic Energy Sciences, U.S. DOE at Oak Ridge National Laboratory under Contract No. DE-AC05-00OR22725 with UT-Battelle, LLC. M.B.N. and J.T.M. are thankful to M. Lazzeri for useful discussions.

\footnotetext{
*kwk@ncsu.edu

${ }^{1}$ K. I. Bolotin, K. J. Sikes, J. Hone, H. L. Stormer, and P. Kim, Phys. Rev. Lett. 101, 096802 (2008).

${ }^{2}$ J.-H. Chen, C. Jang, M. Ishigami, S. Xiao, E. D. Williams, and M. S. Fuhrer, Solid State Commun. 149, 1080 (2009).

${ }^{3}$ M. I. Katsnelson and A. K. Geim, Philos. Trans. R. Soc. London, Ser. A 366, 195 (2008).

${ }^{4}$ E. H. Hwang and S. Das Sarma, Phys. Rev. B 77, 115449 (2008).

${ }^{5}$ F. T. Vasko and V. Ryzhii, Phys. Rev. B 76, 233404 (2007).

${ }^{6}$ R. S. Shishir and D. K. Ferry, J. Phys.: Condens. Matter 21, 344201 (2009).

${ }^{7}$ X. Hong, A. Posadas, K. Zou, C. H. Ahn, and J. Zhu, Phys. Rev. Lett. 102, 136808 (2009).

${ }^{8}$ P. Neugebauer, M. Orlita, C. Faugeras, A.-L. Barra, and M. Potemski, Phys. Rev. Lett. 103, 136403 (2009).

${ }^{9}$ S. Baroni, S. de Gironcoli, and A. Dal Corso, Rev. Mod. Phys. 73, 515 (2001).

${ }^{10}$ S. Piscanec, M. Lazzeri, F. Mauri, A. C. Ferrari, and J. Robert-
}

son, Phys. Rev. Lett. 93, 185503 (2004).

${ }^{11}$ M. Lazzeri, C. Attaccalite, L. Wirtz, and F. Mauri, Phys. Rev. B 78, 081406(R) (2008).

${ }^{12}$ QUANTUM-ESPRESSO is a community project for high-quality quantum-simulation software, based on DFT, and coordinated by Paolo Giannozzi (see http://www.quantum-espresso.org/).

${ }^{13}$ N. Mounet and N. Marzari, Phys. Rev. B 71, 205214 (2005).

${ }^{14}$ H. J. Monkhorst and J. D. Pack, Phys. Rev. B 13, 5188 (1976).

${ }^{15}$ J. L. Mañes, Phys. Rev. B 76, 045430 (2007).

${ }^{16}$ F. Aryasetiawan and O. Gunnarsson, Rep. Prog. Phys. 61, 237 (1998).

${ }^{17}$ This approximation is expected to remain valid in graphene so long as the Fermi level is located below the energy threshold for intervalley scattering processes, which is the case at typical electron concentrations $\left(10^{11}-10^{12} \mathrm{~cm}^{-2}\right)$.

${ }^{18}$ V. Perebeinos and P. Avouris, arXiv:0910.4665 (unpublished).

${ }^{19}$ See, for example, C. Jacoboni and P. Lugli, The Monte Carlo Method for Semiconductor Device Simulation (Springer-Verlag, New York, 1989). 